\title{
El patrón de conducta tipo A y el trabajo. Un estudio sobre satisfacción laboral
}

Raquel Ma García Rodríguez, Sara Ortega Guzmán, Adelaida Gonzales Segura, Eloy López Doblas y Leon Ludwig Lloyd Aldous Kraus. Universidad de Granada

Recepción: 12 de mayo de 2015 | Revisión: 17 de junio de 2015 | Aceptación/Publicación: 17 de julio de 2015

Citar: Garcia, RM., Ortega, S., Gonzales, A., Lopez, E. y Ludwing, L. (2015).El patrón de conducta tipo A y el trabajo. Un estudio sobre satisfacción laboral. ReiDoCrea, 4, 100-105.

Resumen: La presente investigación tiene como objetivo identificar la relación existente entre patrón de conducta tipa A (PCTA) con la satisfacción laboral. Se utilizó para ello una muestra de 57 participantes de las ciudades de Granada y Jaén. Para evaluar el patrón de conducta tipo A y para medir la satisfacción laboral se utilizaron cuestionarios, estos fueron acompañados con una hoja de datos sociodemográficos. El principal resultado nos muestra que no existe una relación estadísticamente significativa entre el patrón de conducta tipo A y la satisfacción laboral. Para mayor detalle y descripción, revisar las conclusiones.

Palabras claves: patrón de conducta tipo A, satisfacción laboral, tipo de trabajo y tipo de contrato.

\section{MANAGEMENT STYLE A AND JOB. A STUDY ABOUT JOB SATISFACTION}

\begin{abstract}
The object of the following investigation is to identify if there is a relationship between Management style A and work satisfaction. In order to determine whether there is a relationship, 57 participants from the cities of Granada and Jaén were interrogated. They were given questionairies to evaluate the Management style $A$ and to measure the level of work satisfaction. They were also handed a sociodemographical data survey. The main result is that there exists no statistically significant relationship between Management style A and work satisfaction. For more details and a broader decription see the conclusions.
\end{abstract}

Key words: Management Style A, Work Satisfaction, type of work, type of contract.

\section{Introducción}

En el análisis psicosocial de los estilos de vida podemos hablar de patrones de conducta que predominan de forma homogénea, independientemente de las diferencias individuales (Mendoza, 1990). Uno de estos es el patrón de conducta tipo A (PCTA) que fue formulado por primera vez por Friedman y Rosenman (1959) y estos lo definieron como un complejo de acción-emoción caracterizado por un impulso continuo para lograr metas autoseleccionadas, pero definidas pobremente, fuerte inclinación a competir, deseos altos de reconocimiento, intento de ser rápido en la realización de sus tareas, implicación en diversas actividades y una gran alerta tanto mental como física. Siguiendo esta línea, Romero y León (1989) destacan que las personas con este tipo de conducta se muestran como trabajadores tenaces que no presentan síntomas de agotamiento, a su vez son muy ambiciosas, agresivas, competidoras, presentan un gran significado del compromiso y la rapidez. La parte negativa de este tipo de conducta puede ser la incapacidad que presentan las personas con conducta tipo A para darse cuenta de sus limitaciones, lo que puede causar algunos fracasos aunque el despliegue de energía sea muy elevado. Otra característica que presenta los sujetos con esta conducta es la acción de responder energéticamente a los estresores ya que tienden a interpretarlos como amenazantes para su autocontrol y equilibrio personal (Rodríguez-Marín, 1995). Glass (1977) indicó que suelen tener un estilo atribucional internalizador, estable y global ante situaciones de frustración, presentando una indefensión aprendida cuando se hace de manera prolongada. Este patrón de conducta se ve relacionado de forma importante con el 
ámbito laboral ya que este es una de las esferas más importantes de la persona debido al gran tiempo que se invierte en él, al gran número de relaciones interpersonales que generan y el gran impacto que tiene en la vida de las personas (Garcia-Sorrentino y Fantin-Salas, 2012). Especialmente se puede relacionar con la satisfacción laboral que según Aldag y Brief (1978) constituye una dimensión actitudinal que ocupa un lugar muy importante en la consideración de las experiencias del humano en el trabajo y se refiere a la conformidad que presenta la persona en relación a su trabajo en sí y al entorno laboral al que pertenece. La satisfacción laboral modula la conducta o comportamiento que el trabajador tiene frente a sus tareas $u$ obligaciones dentro de su trabajo.

Algunos autores han encontrado que el PCTA correlaciona de forma positiva con la insatisfacción laboral, sin embargo Mathieu (1991) nos muestra que el compromiso y la satisfacción laboral están recíprocamente relacionados, no obstante puede que haya variables moduladoras que estén implicadas en estos resultados. También podemos observar que los individuos con un alto compromiso no sufren tanto los resultados negativos en el trabajo, ya que el incremento del estrés solo causa insatisfacción laboral cuando el compromiso laboral es bajo (Begley y Czajka, 1993). De esta forma las personas con PCTA, al tener mayor compromiso con su trabajo, percibirían una mayor satisfacción laboral, con independencia del estrés al que estén sometidos.

A raíz de los estudios anteriores que relacionan los aspectos de satisfacción laboral con mayor compromiso con el trabajo (asociado al PCTA) nuestra hipótesis se basaría en la creencia de que cuanto mayor sean las características del patrón de conducta tipo A en la persona, mayor satisfacción laboral experimentará la misma.

\section{Método}

\section{Participantes}

Para realizar este estudio se ha seleccionado una muestra de 57 personas, las cuales 30 son hombres y 27 mujeres de las provincias de Granada y Jaén, los cuales trabajan 0 han trabajado anteriormente. Las edades de los participantes están comprendidas entre 18 y 58 años con una media de $37(D T=12.86)$ De los sujetos, 3 están desempleados, 20 trabajan a tiempo parcial, 26 a jornada completa y 6 son estudiantes, teniendo a dos participantes que se desconoce esta variable. Por otro lado tenemos que 13 participantes trabajan con contrato indefinido, 17 con contrato temporal, 20 muestran otro tipo de contrato y 7 no rellenaron esta información en el cuestionario. Fueron elegidos de manera aleatoria y participaron en la investigación de manera voluntaria.

\section{Instrumentos}

Los instrumentos utilizados para el estudio han sido dos; en primer lugar la ERCTA (Escala de Retiro de Conducta Tipo A; (Rodríguez, Gil y Martínez, 1996) constituida por ocho items de escala de respuesta tipo Likert graduado con cinco puntos, donde 1 significa "muy bajo" y 5 "muy alto". Esta escala mide la valencia del patrón de conducta tipo A presente en cada individuo, a mayor puntuación obtenida en la escala, mayor presencia de esta conducta en el sujeto (pudiendo categorizarse de forma orientativa como personas tipo A aquellas con unos valores de 24 o superior). La consistencia interna de la escala muestra una fiabilidad de .67 . 
El otro instrumento ha sido el "Cuestionario de satisfacción laboral, S20/23" (Meliá y Peiró, 1998). Dicho cuestionario está compuesto por 23 items de tipo Likert con una escala de respuesta que va desde "muy insatisfecho" (1) a "muy satisfecho" (7) siendo la puntuación de 4 "indiferente". Este es el encargado de medir de una manera útil y rica la satisfacción laboral de las personas teniendo en cuenta las restricciones motivacionales o temporales a las que están expuestos en el contexto del trabajo. Esta es la versión reducida más completa estructuralmente de las desarrolladas a partir del S4/82. La fiabilidad de este cuestionario es de .92.

A su vez se les pedía a los participantes algunos datos socio-demográficos entre los que aparecen el sexo, la edad, la nacionalidad, el tipo de trabajo y el contrato que tienen en su empleo.

\section{Procedimiento}

Para la cumplimentación de los instrumentos los evaluadores se desplazaron hasta los domicilios de los participantes, siendo un día del fin de semana para no encontrarlos en su lugar de trabajo. Cada evaluador llevaba consigo un cuadernillo impreso para cada participante, junto con una hoja de consentimiento informado la cual debían de firmar antes de comenzar, dando la opción de retirarse en cualquier momento. Todos los participantes recibieron información general sobre la investigación (la relación entre ciertos aspectos y la satisfacción en el trabajo), sin relevar mucha información directamente relacionada con los objetivos de la misma. El orden para completar los cuestionarios fue en todos los casos el mismo. Además se le dio la opción de contactar con el evaluador tanto por vía telefónica como por correo en el caso de tener alguna duda o querer más información acerca de la investigación.

\section{Resultados}

\section{Análisis de Datos}

Con objetivo de analizar los datos de la muestra se ha procedido a realizar un análisis con el programa SPSS 20.0 con el cual hemos obtenido información acerca de diversas variables como son edad, sexo, tipo de trabajo o contrato de los participantes y de las dos variables principales en el estudio, patrón de conducta tipo $A$ y satisfacción laboral.

Para comenzar podemos decir que la media de puntuación en el cuestionario de patrón de conducta tipo A es aproximadamente normal, con una puntuación media de 29.77 (sobre una media posible de 40) que coincide con la mediana que en este caso sería 30 y presenta una desviación típica de 4.47. Teniendo en cuenta estudios anteriores (Miller, Turner, Tindale, Posavac, y Dugoni, 1991) indican que alrededor del $50 \%$ de la población presenta PCTA por tanto la puntuación de 24 se puede considerar orientativa. Por otro lado la media de satisfacción laboral sería 108.06 (sobre un total posible de 161) con una mediana de 106.50 y una desviación típica de 23.64 .

Para comprobar la hipótesis acerca de la relación entre PCTA y satisfacción laboral se realizó un análisis de correlación de Pearson, cuyos datos no mostraron relación entre ambas variables $(r=.18, p=.20)$. También se correlacionaron ambas con la variable edad. La correlación entre PCTA y edad tampoco mostró una corrrelación estadísticamente significativa $(r=-.14, p=.29)$. La correlación entre satisfacción laboral y edad sí que mostraba cierta relación (marginalmente significativa) $(r=.35, p$ $=.081)$. 
Con el objetivo de comprobar si existen diferencias en el PCTA en función del tipo de trabajo, se realizó un ANOVA de un factor. Los resultados obtenidos se muestran en la Gráfica 1, donde se aprecia que la mayor media la presentan los individuos con trabajo a tiempo parcial $(M=31.10)$ en comparación a los desempleados que son los que puntúan más bajo $(M=28)$. A pesar de estas diferencias, podemos afirmar que el tipo de trabajo no es esencial para determinar el patrón de conducta ya que las diferencias mostradas no son estadísticamente significativas, con un grado de significación 0.374 .

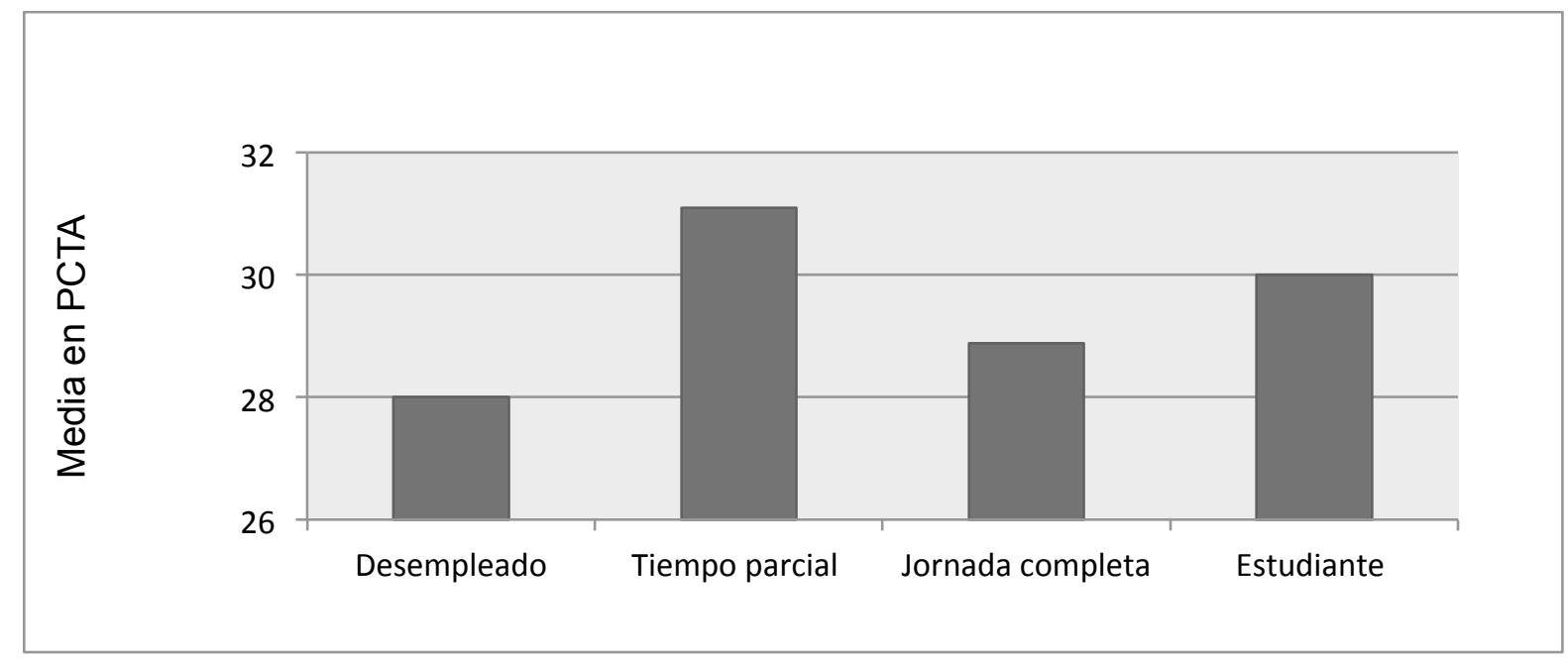

Gráfica 1. Nivel de PCTA en función del tipo de trabajo

La Grafica 2 nos muestra la media de satisfacción laboral en relación con el tipo de trabajo que realizan los participantes, así contemplamos que en este caso la mayor puntuación se da en personas que tienen trabajo a jornada completa $(M=109.77)$ seguido muy de cerca por el empleo a tiempo parcial $(M=108.62)$, siendo los estudiantes los que están en el último lugar $(M=95.20)$. Las diferencias en este caso tampoco son significativas, con un grado de significación de 0.602 .

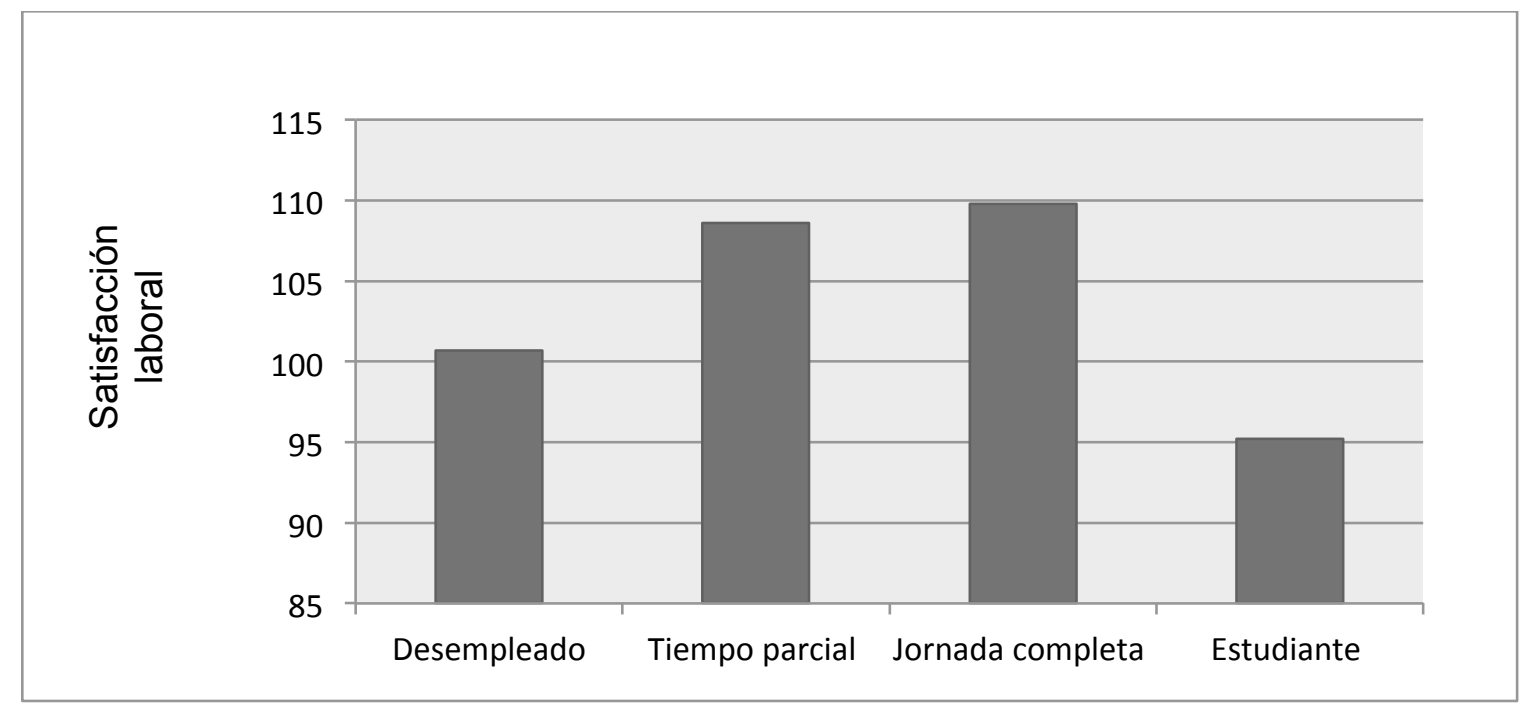

Gráfica 2. Grado de satisfacción en función del tipo de trabajo

Para comparar nuestras variables PCTA y satisfacción laboral con la variable tipo de contrato utilizamos también la prueba $T$. Los resultados que se muestran en la Tabla 1 indican que la media de sujetos con contrato a tiempo parcial $(M=31.1)$ también es 
similar a la media de sujetos con contrato a jornada completa $(M=28.9)$, y lo mismo ocurre con las medias de contrato a tiempo parcial $(M=108.6)$ y las de contrato a jornada completa $(M=109.8)$ respecto a la satisfacción laboral.

\begin{tabular}{|cccc|}
\hline \multicolumn{5}{c}{ Tabla 1 } \\
Tipo de contrato en relación a las variables PCTA & y satisfacción laboral \\
\hline & \multicolumn{4}{|c|}{ Tipo de contrato } & Media & Desviación típica \\
\hline \multirow{2}{*}{ PCTA } & Tiempo parcial & 31.100 & 4.229 \\
& Jornada completa & 28.885 & 5.458 \\
& & & \\
\multirow{2}{*}{ SL } & Tiempo parcial & 108.625 & 25.917 \\
& Jornada completa & 109.792 & 24.194 \\
\hline
\end{tabular}

Para comparar nuestras variables PCTA y satisfacción laboral con la variable sexo utilizamos una prueba $T$. Los resultados que se muestran en la Tabla 2 indican que la media de los hombres $(M=29.67)$ y la de las mujeres $(M=29.89)$ son similares para el PCTA, al igual que ocurre con las medias de hombres $(M=107.74)$ y mujeres $(M=$ 108.43) respecto a la satisfacción laboral.

\begin{tabular}{|llcc|}
\hline \multirow{4}{*}{ PCTA y satisfacción laboral en relación a la variable sexo } \\
\hline & Sexo & Media & Desviación típica \\
\hline \multirow{3}{*}{ PCTA } & Hombre & 29.666 & 3.933 \\
& Mujer & 29.889 & 5.084 \\
& & & \\
\multirow{2}{*}{ SL } & Hombre & 107.741 & 22.898 \\
& Mujer & 108.434 & 24.994 \\
\hline
\end{tabular}

La prueba $T$, nos muestra, al igual que el ANOVA realizado anteriormente que no hay ninguna diferencia significativa teniendo en cuenta el tipo de trabajo, independientemente de analizar los cuatro o solo los dos que presentan más sujetos.

\section{Discusión}

El objetivo de este estudio ha sido analizar la relación existente entre el patrón de conducta tipo A y la satisfacción laboral, así como la influencia de otros factores en la satisfacción laboral (sexo, edad, tipo de contrato y tipo de trabajo).

Los resultados de este estudio entre los constructos PCTA y satisfacción laboral en una muestra de personas que actualmente trabajan o que han trabajado anteriormente indican que no existe correlación entre las variables PCTA y satisfacción laboral. De modo que no podemos confirmar nuestra hipótesis. Sin embargo otros autores como Begley y Czajka, (1993) encontraron que las personas con PCTA sufren menos los resultados negativos en el trabajo, mostrando una mayor satisfacción laboral.

Para comparar si existen diferencias entre las distintas variables que se han intentado estudiar en esta investigación, se ha llevado a cabo un análisis de la varianza. Estos grupos comparados son el tipo de trabajo y contrato. Con el tipo de trabajo y en primer lugar con el PCTA, si nos basamos en estos resultados, podemos decir que las personas con un contrato de trabajo a tiempo parcial presentan más PCTA lo que no concuerda con lo que actualmente se sabe que la conducta tipo A está relacionada con largas horas de trabajo (Sorensen et al., 1987). Los desempleados presentan las 
puntuaciones más bajas de PCTA. Con la satisfacción laboral cabe destacar que las personas con un contrato a jornada completa son las más satisfechas, seguidas de las que tienen un contrato a tiempo parcial. Los estudiantes sería el grupo más insatisfecho. También se ha comprobado el papel modulador del sexo con nuestras dos variables principales. Según nuestros resultados, no hay diferencias en el PCTA entre hombres y mujeres al igual que ocurre con la satisfacción laboral.

Sin embargo todos estos resultados no son significativos, por lo que sólo nos pueden servir de orientación en cuanto a las relaciones entre los constructos y las diferentes variables que hemos seleccionado para nuestro estudio.

No obstante, los resultados encontrados en este estudio se deben considerar teniendo en cuenta algunas limitaciones. Por ejemplo, las de tipo muestral, ya que la que se ha seleccionado no es estadísticamente significativa $(\mathrm{N}=57)$, esta puede ser una de las posibles causas por la que los resultados de nuestra investigación no hayan sido significativos. Otra posible limitación puede ser la de la distribución de los sujetos entre las variables tipo de contrato y tipo de trabajo pues puede que no haya sido homogénea. Una tercera limitación encontrada es la baja fiabilidad del cuestionario para medir el PCTA utilizado. Por lo tanto para futuros estudios en este ámbito tendrían que tenerse en cuenta y controlarse estos tres aspectos.

\section{Bibliografía}

García, M. A. y Berrios, M.P. (1999). El significado del trabajo en personas con patrón de conducta tipo A. Psicothema, $11(2), 357-356$.

Hernández, L. y Olmedo, E. (2004). Un estudio correlacional acerca del síndrome "estar quemado" (Burnout) y su relación con la personalidad. Apuntes de Psicología, 22(1), 121-136.

López, B., Osca, A. y Peiró, J.M. (2007). El papel modulador de la implicación con el trabajo en la relación entre el estrés y la satisfacción laboral. Psicothema, 17 (1), 81-87.

Rodriguez, C., Gil, P. y Martínez, R. (1996). Presentación de la escala de Retiro de Patrón de Conducta tipo A (ERCTA). Psicothema, 8(1), 207-213.

Meliá, J. L. y Peiró, J.M. (1989). La medida de la satisfacción laboral en contextos organizacionales: El Cuestionario de Satisfacción S20/23. Psicologemas, 5, 59-

74.

De la Fuente, J. y De la Fuente, M. (1995). Análisis componencial del Patrón de Conducta tipo A y respuestas ansiógenas situacionales especificas: implicaciones para la intervención. Psicohema. 7 (2), 267-282.

Berrios, M. y García, J. (2006). Efecto de la congruencia entre el patrón de conducta tipo A y el tipo de tarea en el rendimiento y la satisfacción. Revista Latinoamericana de Psicología, 38 (2), 271-284.

García, H. y Fatín, M. (2014). Patrón de conduta tipo A en trabajadores de la ciudad de San Luis, su relación con actitudes disfuncionales. Journal of Behavior, Health \& Social Issues, 5(2), 31-42.

Edmans, A., Li, L. y Zhang, C. (2014). Employee Satisfaction, Labor Market Flexibility, and Stock Returns Around The World. ECGI Working Paper Series in Finance, 433, 1-47.

Gil, M. e Izquierdo, A. (2004). Intervención en el Patrón de Conducta Tipo A. Un modelo interdisciplinar. Persona, 7, 7185. 